\title{
DIGITAL TECHNOLOGY Surgery in the 2020s: Implications of advancing technology for patients and the workforce
}

\author{
Author: Richard SC Kerr ${ }^{\mathrm{A}}$
}

As the surgical workforce, surgical techniques and patient expectations change, the Royal College of Surgeons of England is actively engaged in taking forward the recommendations of its Future of Surgery Commission. Here the commission's chair articulates the implications for smaller hospitals and the need for achieving interoperability and safe sharing of patient data across different systems, so enabling immediate access to patients' records across healthcare organisations; extension of regulation to surgical care practitioners, reflecting the recent decision to regulate physician associates and physician assistants; introducing a UK-wide registry of surgical devices, with tracking for implantable devices; implementing a robotics strategy to help the NHS plan and purchase new surgical robotics, as well as monitor their use and the effect on outcomes; and investing in genomic medicine and artificial intelligence for diagnostics, and in stem-cell research for treatment.

KEYWORDS: Surgery, robotics, artificial intelligence, genomics, training

\section{Introduction}

One of the biggest ongoing debates in surgery is the extent to which complex medical interventions should be centralised to improve outcomes. For the NHS, 'big' has often meant better, and more specialised. For patients, where their surgery takes place can make the difference between feeling relatively calm about a daunting prospect, or causing intense worry by adding distance from relatives during recovery. This can be particularly important for older people who may be too frail to travel long distances, as well as for people on low incomes and those with caring responsibilities. Older people are also more likely to live outside metropolitan areas and this trend is set to increase.

In 2017, the Royal College of Surgeons of England (RCS) sponsored an independent commission on the future of surgery, which I chaired. While some of the technological developments covered by our report will require 'centres of excellence' to get

Author: ${ }^{\text {A }}$ neurosurgeon and council member, Royal College of Surgeons of England, London, UK them off the ground, the long-term trajectory is towards more care being delivered locally.

Our commission's final report drew on a comprehensive literature review, more than 70 contributions of written evidence, and 4 days of oral evidence. It covered likely changes to the surgical workforce and how they are trained; artificial intelligence (AI) and genomics in diagnostics; 3D printing and robotic assistance in operating theatres; and the regulatory reforms which are needed to keep pace.

A report published in 2016, looking at the potential for automation across different sectors found that health and education are the areas where machines are least likely to take over from humans. ${ }^{1}$ Applied expertise and the crucial need for human interaction will never, in all probability, render clinicians obsolete. Although AI will increasingly be used to find solutions to specific replicable problems - most particularly in diagnosis it seems highly unlikely that it could replace surgeons in the foreseeable future. A surgical robot able to perform an entire surgical procedure autonomously and handle the unpredictability of its possible complications is a very distant possibility. Instead, robotics in the operating theatre represent highly complex and sophisticated tools for surgeons and their teams to use, not substitutes for their presence.

\section{Robotics}

Contrary to the tenor of some of the news coverage, surgical robots in operating theatres are not new. There are already 115 in action in the UK. On mainland Europe, the concentration is greater still at up to three robots per million people in the population. To ensure that the technology is sensibly deployed, there is an urgent need for the NHS to map where robots already are, and to plan for the expansion of their use.

Thus, one of our commission's key recommendations is the development of a robotics strategy for the NHS to manage their proliferation. This is of special relevance to smaller hospitals, who may particularly wish to have the more portable types of surgical robot, at their disposal. In future, it may be robots, rather than patients, that have to travel for operations. Such a strategy would identify not just the potential capital funding for buying more robotic equipment, but also enable development of high-quality training programmes to ensure they are used safely.

Robotics are developing so fast that, in most cases, robot manufacturers will be best placed to train surgeons in their use. Those very companies, however, cannot be both gamekeeper and 
poacher, providing the training and also certifying its quality. The RCS is therefore discussing how it might provide accreditation to industry training, with clear requirements and independent assessment of the training portfolio. Whether provided by robotics manufacturers or academic institutions, it is very clear that training programmes must involve whole surgical care teams, including all those involved in delivering that episode of care.

We are keen, too, to see robotics built into routine training as surgeons progress through their specialty trainee grades; this will mean everything from making the subject part of the existing 'boot camps' that students go through to setting up training fellowships. We are looking to the industry to participate in making these things happen, and urging medical schools to address them as integral parts of the future curriculum.

One of the obstacles to the advancement of robotic surgery is cost. There is a good opportunity for networks of hospitals including smaller hospitals - to work together in sharing this resource. China has already demonstrated the potential of remote surgery, where a specialist conducted a whole procedure on a patient from thousands of kilometres away through remote robotic assistance over $5 \mathrm{G}$ networks. ${ }^{2}$ We are possibly some way from safe, routine use of such technology on patients in the UK, but the use of augmented reality (AR) images to transmit real-time information to a specialist surgeons at another location, to seek their guidance in completing a complex procedure, is already with us.

\section{D printing}

$3 \mathrm{D}$ printing is another technology set to transform both planning for surgery, and the range and effectiveness of implants available. 3D printing software can be used to extract data from patient imaging technologies and print personalised and custom-made guides for surgery and implants to replace or hold in place body parts or anatomical structures affected by diseases. 3D planning and printing technologies are likely to become widely available, and the printed products are robust enough to be transferred to all hospitals for surgical teams locally to use.

These developments could make surgery safer and more precise, and they open avenues for surgical procedures that are currently too complex or have poor outcomes. Such innovation should lead to an increase in patient choice, expectations and a demand for bespoke care. The provision of 3D models to patients could improve patient understanding of procedures they are about to undergo, and so facilitate more informed consent. In view of the 2015 Montgomery judgment, these additional tools to ensure that patients know as much as possible about their operations, and the material risks, are a potentially critical tool for doctors. ${ }^{3}$

\section{Stem cells}

A further rapidly developing research and treatment area is the use of stem cells. Such treatments already being used by the NHS, bone marrow stem cell transplants for, among other things, leukaemia and sickle cell anaemia, though the existing risks of a bad reaction between host and graft (donor) cells has kept the procedure from becoming routine. ${ }^{4}$ Gene-modified bone marrow stem cell transplants have been used recently at Great Ormond Street Hospital to treat very sick children suffering from severe disorders of the immune system. ${ }^{5}$

Ophthalmologists are leading the way in developing the science further. In particular, Prof Robin Ali, an Academy of
Medical Sciences fellow at Moorfields Eye Hospital, has helped pioneer clinical trials using stem cell transplants to treat macular degeneration have shown promise, noticeably improving vision in a number of participants. ${ }^{5}$ Prof Robert MacLaren, at the Oxford Stem Cell Research Institute, is also engaged in a programme of research to identify areas of stem-cell and regenerative medicine which could develop new treatments for retinal disease.

Further afield, doctors at Osaka University in Japan restored a patient's sight in one eye by - for the first time - transplanting corneal tissue created from stem cells. While the only treatment available to people in the UK today is a corneal transplant from a deceased person, this new procedure could enable healthy, live donors to give their cells for transplantation. It is hoped that rejection rates will be far lower too; whereas $20 \%$ of transplant patients react against their new cornea, the stem cells take on the characteristics of the host body as they develop. ${ }^{6}$

Stems cells could go on to be able to treat and prevent conditions like Parkinson's, Alzheimer's, heart disease and arthritis. Spinal cord cells may one day restore mobility to those who have been profoundly paralysed, and skin cells could help burn victims recover.

\section{Patient-facing technology}

Though the most exciting and sophisticated advances are in operating theatres, relatively simple smartphone technology is set to have a positive impact on patients before and after operations take place. Already the NHS app enables people to book simple general practitioner (GP) appointments online. Meanwhile the GoodSAM app commissions 'smartphone activated medics' through a system which integrates with ambulance service dispatch systems to trigger a response from nearby, trained, bystanders while an ambulance is on route.

In future, postoperative monitoring will be able to take place remotely with the patient showing doctors how their wounds are healing using their own smartphone camera. Smart watches can monitor pulse - and of course many people already collect data on their heart rate, their calorie consumption and their exercise through such devices. For more complex data collection, short-term wearable, implantable or ingestible sensors could help monitor vital signs and detect postoperative infections perhaps by detecting bacteria in a wound, and checking the body's core temperature.

\section{Training and future surgical team}

As science advances with new procedures, we will come to rely on ever more sophisticated technology to support training for surgeons in carrying them out. The days of "watch one, do one, teach one' as a hierarchy for training in surgical procedures are rightly gone for good. New portable virtual reality (VR) and AR systems will be able to be placed not just in major institutions but in smaller hospitals to enable coordinated teaching alongside clinical practice. There is a distinct shift now from static anatomical displays - which were limited in scope to rigid bone structures in orthopaedic and neurosurgery - to dynamic displays which accurately simulate live tissues. Gynaecological, colorectal and cardiothoracic patients will be the next to benefit from these developments, and they can be deployed not just in specialist teaching hospitals but anywhere. Across the NHS, we will see AR guiding and training surgeons in their work.

The rapid changes awaiting the health service mean that the path of a surgeon qualifying today will be very different to my own 
surgical career. Alvin Toffler, in his book Future shock predicted that 'the illiterate of the 21st century will not be those who cannot read or write but those who cannot learn, unlearn and relearn'. ${ }^{7}$ That capacity to keep up with changing times will be a critical part of the skill set required for surgeons in future.

The James Robertson Justice 'surgeon master' model of management is long passed. More than ever, we will be active participants and leaders, not just in our clinical fields but of surgical teams. These will include a whole range of healthcare professionals, who must be appropriately regulated and may well in the future be able to perform some parts of operations, or even whole procedures under supervision.

Alongside new and greater expectations of flexibility and adaptability from the NHS and wider healthcare sector should come new expectations of flexibility and adaptability from the surgeons and their teams. There was a great deal of synergy in the findings of our Future of Surgery report and that of the extensive Topol review into Preparing the healthcare workforce to deliver the digital future. ${ }^{8}$ Both reports recognise that expectations around work-life balance are rightly shifting, and that the development of new technology, which could reduce workloads, comes alongside ever increasing demand for services.

While journalists reasonably like to speculate about a future in which 'Johnny 5' style robots will meet patients at the hospital door and perform everything from making tea to advanced neurosurgery, the reality is very different. Emerging technology is there to support the clinical workforce, and provide better or quicker outcomes for patients. The future is of a flexible workforce assisted by technology, not to reduced workforce replaced by it. Our clinical relationships with patients will continue to be of paramount importance. Compassion, empathy and the human touch cannot be simulated.

\section{Prevention is better than cure}

Technological advances are not just about treatment, but about prevention. The Lancet Digital Health recently published a review of 20,500 articles on the capacity of artificial intelligence to make accurate diagnoses. The limits on the evidence available were exposed by the process, which found that only 14 of the studies concerned directly compared the performance of AI with that of healthcare professionals. These 14 did nonetheless conclude that deep learning algorithms, which use thousands of medical images to identify patterns of disease, can make correct diagnoses in $87 \%$ of cases, compared to $86 \%$ by healthcare professionals. Similarly, the algorithms can exclude $93 \%$ of patients who don't have disease, compared to $91 \%$ among healthcare professionals. ${ }^{9}$

The implications for hospitals are clear. Where a patient might previously have had to visit a specialist for a diagnosis, their tests will instead be able to be analysed locally and a diagnosis delivered instantly. The use of machine learning will, in future, be combined with the insights available from genomics and from electronic health records to improve our understanding of disease profiles both across whole populations and, then hopefully, in individual patients.

Despite the travails of NHS IT projects like care.data, government must once again address the issue of safe, consented and transparent data sharing. For our health service to be truly national, professionals in every part of it - from a remote GP surgery to the big city, multidisciplinary centres of excellence must be able to access data on patients in their care. The Department of Health and Social Care has set out some welcome guiding principles for making this happen - user need, privacy and security, interoperability (compatibility of data), and openness and inclusion - but these now need to be put to work on the ground. ${ }^{10}$

With anonymised information, gathered from big data sets, we should be able to predict which parts of the population are most at risk, influencing screening and surveillance, and early treatment. Liquid biopsies with analysis of fragments of tumour deoxyribonucleic acid found in peripheral blood, for example, will not only enable early diagnosis of cancer and other tumours but allow monitoring for treatment effectiveness and recurrence. The tests will be able to be conducted at GP surgeries or local hospitals, without recourse to a specialist centre.

Genomics, too, will play a huge part in the future improvement of care delivered to patients.

Many conditions result from a major inherited genetic predisposition, including cancer, arthritis, heart disease and diabetes. Prevention, prediction and early diagnosis of disease driven by genomic analysis will influence the type of surgery undertaken, and may even reduce the amount of surgery required. The direction of travel is towards organ sparing surgery, with diagnosis at an earlier stage of disease. However, earlier diagnosis does come with potential pitfalls. Patients could find themselves 'over-treated' with risk-reduction surgery offered to those who would not otherwise have needed any medical intervention. Estimating and contextualising risk accurately will be of greater importance than ever.

Herein therefore lies an opportunity for the NHS, which is uniquely placed to gather data on the efficacy of new technology. Unlike other healthcare systems elsewhere in the world, there is a degree of central control and oversight. Core to that oversight should be a comprehensive registry of surgical devices and procedures - from robots to the products we implant, like mesh - which can be used to track who has had what procedure, delivered in what way, using what surgical products. The government is presently awaiting the outcome of the eagerly awaited Cumberlege review into the use of surgical mesh in uro-gynaecology; hopefully the outcome will cover more than just mesh used in this situation.

\section{Conclusions}

As the surgical workforce, surgical techniques and patient expectations change, so must regulation of the sector. The RCS is actively engaged in taking forward the recommendations of our Future of Surgery Commission, which I have considered here and in summary include:

> achieving interoperability and safe sharing of patient data across different systems, enabling immediate access to patients' records across healthcare organisations

> extension of regulation to surgical care practitioners, reflecting the recent decision to regulate physician associates and physician assistants

> introducing a UK-wide registry of surgical devices, with tracking for implantable devices

> implementing a robotics strategy to help the NHS plan and purchase new surgical robotics, as well as monitor their use and the effect on outcomes

> investing in genomic medicine and AI for diagnostics, and in stem-cell research for treatment.

For patients, technological developments all promise the potential of better outcomes and, in places, more local treatment. The potential of innovation must always be held up to the light 
of rigorous research, testing and monitoring. However, on the whole, patients should be able to expect less invasive, and more accurate surgery in future, with faster recovery times and smaller differences in performance between surgeons.

Earlier intervention may mean fewer operations for some conditions, and earlier operations for others - potentially avoiding, for example, distressing chemotherapy and radiotherapy in cancer patients by the early complete removal of tumours. Improvements to surgical techniques, and greater consistency in outcomes, will also enable us to offer operations to older, more frail patients than we have in the past. Diagnosis and treatment should often be able to be delivered nearer to where patients already are, at hospitals in or near their own community. The most welcome overall development that flows from new technology is that the NHS is in so many respects set to become truly a 'health' service, about diagnosing and preventing illness rather than treating those who are already sick.

\section{References}

1 Chui M, Manyika M, Miremadi M. Where machines could replace humans - and where they can't (yet). McKinsey Quarterly 2016.

2 Loeffler J. China performs country's first ever $5 \mathrm{G}$ remote brain surgery. Interesting Engineering 2019.

3 Chan SW, Tulloch E, Cooper ES et al. Montgomery and informed consent: where are we now? BMJ 2017;357;j2224.
4 NHS. Sickle cell disease. NHS, 2019. www.nhs.uk/conditions/sicklecell-disease/treatment

5 Lechler R. What does the future hold for stem cell treatments? Academy of Medical Sciences, 2018. https://acmedsci.ac.uk/more/ news/what-does-the-future-hold-for-stem-cell-treatments-

6 Lloyd Parry R. Woman regains sight after corneal transplant from stem cells. The Times 2019. www.thetimes.co.uk/article/womanregains-sight-after-corneal-transplant-from-stem-cells-9jgmxs9n6\#

7 Toffler A. Future shock. London: Random House, 1970.

8 Topol E. Preparing the healthcare workforce to deliver the digital future. London: Health Education England, 2019.

9 Liu X, Faes L, Wagner SK et al. A comparison of deep learning performance against health-care professionals in detecting diseases from medical imaging: a systematic review and meta-analysis. Lancet Digital Health 2019;1:271-91.

10 Department for Health and Social Care. Creating the right framework to realise the benefits for patients and the NHS where data underpins innovation. Department for Health and Social Care, 2019.

Address for correspondence: Mr Richard Kerr, c/o Policy Team, Royal College of Surgeons of England, 35-43 Lincoln's Inn Fields, London WC2A 3PE, UK.

Email: policy@rcseng.ac.uk

\section{Consent and confidentiality in genomic medicine}

Genetic or genomic tests are increasingly used in everyday medical practice. Every clinical field will encounter such tests to a greater or lesser extent.

Published in 2019, this third edition by the Joint Committee on Genomics in Medicine provides updated guidance on the use of genetic and genomic information in the clinic. Health professionals from all areas of medicine need to know and understand how consent and confidentiality issues may arise, and to understand the potential ways in which the use of genomic tests may change the nature of the relationship between healthcare professionals and patients.

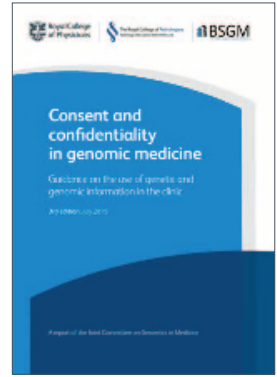

Download the guidance at: www.rcplondon.ac.uk/consent-confidentiality-genomic-medicine 